\title{
Género e internacionalización: un estudio empírico sobre empresas asentadas en Canarias
}

\author{
Antonia M. García-Cabrera ${ }^{1}$, Sonia M. Suárez-Ortega, Francisco J. Gutiérrez-Pérez, María \\ José Miranda-Martel, Sara Ojeda-González, Arístides Olivares-Mesa
}

Universidad de Las Palmas de Gran Canaria, España

doi: https://doi.org/10.20420/eni.2021.452

\begin{abstract}
Resumen
Este artículo sintetiza diversos hallazgos obtenidos en un estudio realizado por el Grupo de Investigación "Estrategia y Negocios Internacionales" de la Universidad de Las Palmas de Gran Canaria y financiado por la Sociedad Canaria de Fomento Económico (PROEXCA). El estudio analiza los posibles nexos entre el género de los directivos y la internacionalización empresarial. Tomando una muestra de 153 empresas canarias internacionalizadas (o con potencial para ello), los resultados revelan que, si bien el género del decisor no condiciona el interés de la empresa por internacionalizarse, la mayor proporción de mujeres en el equipo directivo sí incrementa dicha actividad internacional.
\end{abstract}

Palabras clave: desigualdad de género, internacionalización empresarial, mujeres, Canarias.

Clasificación JEL: J16, M16.

Agradecimiento: Los autores agradecen el encargo recibido de la Sociedad de Sociedad Canaria de Fomento Económico, S.A. (PROEXCA) [Contrato 240.013.0366].

Fuente de referencia: Contrato suscrito en diciembre de 2020 entre PROEXCA y la Fundación Universitaria de Las Palmas (FULP) en representación del Grupo de Investigación Estrategia y Negocios Internacionales de la ULPGC para la realización del proyecto "Análisis del número de mujeres que tienen cargos en empresas internacionales en canarias, así como del número de directivas y su comparativa con el número de hombres".

\section{Introducción}

De acuerdo con la literatura académica reciente sobre gestión de empresas podría afirmarse que, en general, las mujeres participan menos que los hombres en las actividades relativas a la internacionalización empresarial. Estudios previos analizan esta desigualdad a partir de bases de datos internacionales, las cuales permiten realizar comparaciones estadísticas entre países pero que no contribuyen a una profunda comprensión de las causas de dicha desigualdad dentro de cada país (Eden \& Gupta, 2017). Sin embargo, identificar y comprender estas causas resulta esencial para la formulación de políticas y estrategias que, desde el ámbito local o regional, contribuyan de manera efectiva a superar la desigualdad de género.

En este contexto, en diciembre de 2020, la Sociedad Canaria de Fomento Económico SA $(\text { PROEXCA })^{2}$ y la Fundación Canaria Universitaria de Las Palmas (FULP) firmaron un contrato destinado a que el Grupo de Investigación "Estrategia y Negocios Internacionales" (ENI) analizase inicialmente cuántas mujeres ocupan puestos en empresas canarias internacionales, así como el número de mujeres directivas y la comparación con el número de hombres.

Tal y como muestra la figura 1, este estudio se enmarca en un contexto complejo que tiene su

\footnotetext{
${ }^{1}$ Autor de correspondencia: antonia.garcia@ulpgc.es.

${ }^{2}$ Empresa pública del Gobierno de Canarias cuya finalidad es promover la internacionalización de las empresas canarias y atraer inversiones estratégicas hacia esta región.
} 
origen en tendencias supranacionales, nacionales y regionales, relacionándose:

- A nivel supranacional, con los Objetivos de Desarrollo Sostenible (ONU) de Naciones Unidas (2015), específicamente en el Objetivo 5 y el indicador 5.5.2.

- A nivel nacional español, con dos leyes y el Plan de Acción para la internacionalización de la economía española 2021-2022, en los que se establece la igualdad de género como uno de los cuatro pilares para transformar la economía en su conjunto.

- A nivel autonómico, con el objetivo del Gobierno canario sobre la diversificación de la economía regional mediante su internacionalización.

\section{Objetivos y metodología de la investigación}

Ante la escasez de referencias previas similares, este estudio perseguía dos objetivos principales: (1) establecer las bases para comprender las posibles desigualdades entre mujeres y hombres en el ámbito de la internacionalización empresarial en Canarias, y (2) ofrecer a las administraciones públicas información útil para el diseño de programas que promuevan la participación de mujeres y hombres en proyectos de internacionalización.

Debido a la falta de información fiable y actualizada sobre el número de empresas canarias internacionalizadas y, en consecuencia, la compleja definición del universo, la población objetivo de este estudio fue estimada a partir de dos tipos de fuentes:

Figura 1. Relación del estudio con el contexto regional, nacional e internacional

\section{Ámbito supranacional}

(2) OBJETIV

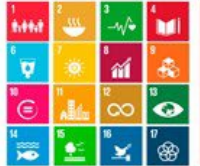

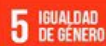
$\theta$
Objetivo 5. Lograr la igualdad entre los géneros y empoderar a todas las mujeres y las niñas
Meta 5.5: Asegurar la participación plena y efectiva de las mujeres y la igualdad de oportunidades de liderazgo a todos los niveles decisorios en la vida política, económica y pública

Indicador 5.5.2: Proporción de mujeres en cargos directivos

\section{Ámbito nacional (España)}

Ley Orgánica 3/2007, de 22 de marzo, para la igualdad efectiva de mujeres y hombres

Real Decreto-ley 6/2019, de 1 de marzo, de medidas urgentes para garantía de la igualdad de trato y de oportunidades entre mujeres y hombres en el empleo y la ocupación

Plan de Acción para la Internacionalización de la Economía Española 2021-22

\section{Ámbito regional (Canarias)}

Objetivo principal del gobierno canario: La diversificación de la economía regional, estrechamente relacionada con el éxito de la internacionalización de las empresas canarias 
- Una base de datos de 1.590 empresas canarias con actividad internacional y que presentan sus cuentas anualmente en el Registro Mercantil, obtenida de Informa D\&B.

Y una base de datos ad hoc sobre empresas canarias internacionalizadas o con potencial de internacionalización desarrollada por el propio Grupo ENI a partir de 33 recursos de información secundaria (directorios de Africainfomarket, Clústeres, Espacios Industriales, etc.). En total, se identificaron 2.283 negocios.

Al fusionar ambas bases de datos y una vez eliminadas las referencias duplicadas, la población objetivo quedó finalmente formada por un total de 3.462 empresas canarias.

Al mismo tiempo, se desarrolló un cuestionario para indagar en los posibles antecedentes y consecuencias de la participación de la mujer en la internacionalización de las empresas canarias. Este cuestionario fue diseñado con la finalidad de ser respondido por el responsable de la toma de decisiones asociadas a la internacionalización de la empresa (en adelante, el decisor) y constaba de 53 preguntas distribuidas en seis bloques:

1. Empresa y cargo actual.

2. Formación y experiencia profesional.

3. Rasgos y características en el desempeño profesional.

4. Actividades internacionales de su empresa.

5. Equilibrio entre el trabajo y la vida privada.

6. Perfil demográfico.

Tras la realización del trabajo de campo, llevado a cabo entre el 20 de mayo y el 16 de julio de 2021, se recabaron 153 cuestionarios válidamente cumplimentados (la tabla 1 resume los aspectos metodológicos de este estudio).
Tabla 1. Ficha técnica del estudio

\begin{tabular}{|c|c|}
\hline Tipo de investigación & Cuantitativa \\
\hline Ámbito & Regional \\
\hline Universo & $\begin{array}{l}\text { Empresas instaladas en Canarias, } \\
\text { internacionalizadas o con } \\
\text { potencial para internacionalizarse }\end{array}$ \\
\hline $\begin{array}{l}\text { Fecha de trabajo de } \\
\text { campo }\end{array}$ & $\begin{array}{l}\text { Del } 20 \text { de mayo al } 16 \text { de julio de } \\
2021\end{array}$ \\
\hline Tamaño muestral & 157 (cuestionarios válidos: 153) \\
\hline Error muestral & $5,59 \%$ \\
\hline $\begin{array}{l}\text { Instrumento de } \\
\text { medición }\end{array}$ & $\begin{array}{l}\text { Encuesta online estructurada ( } 52 \\
\text { preguntas) }\end{array}$ \\
\hline Software estadístico & SPSS Statistics \\
\hline Técnicas estadísticas & $\begin{array}{l}\text { - Análisis de frecuencias } \\
\text { - Medidas de centralización y } \\
\text { dispersión } \\
\text { - Coeficientes de variación } \\
\text { - Contraste de hipótesis: t-test de } \\
\text { diferencias de medias y } \\
\text { ANOVA } \\
\text { - Tablas de contingencia y test } \\
\text { Chi-cuadrado }\end{array}$ \\
\hline
\end{tabular}

\section{Resultados}

\section{La empresa y su expansión internacional}

De las 153 empresas participantes en el estudio, el $62,8 \%$ afirma tener experiencia en mercados internacionales $y$ otro $22,2 \%$ manifiesta estar interesada en acometer actividades de emprendimiento internacional. $\mathrm{La}$ actuación de la empresa en los mercados exteriores se caracteriza por:

- Relativa importancia del peso de la facturación internacional de las empresas analizadas. Para el 50,6\% los mercados exteriores suponen $1 / 4$ de su facturación total, mientras que otro $21,7 \%$ obtiene toda su facturación en el exterior.

- Diversidad de destinos en las exportaciones de servicios (ej. EE.UU., Marruecos, Mauritania, Cabo Verde y Reino Unido) y en las exportaciones de productos (ej. Alemania, Italia, Mauritania, Francia, EE.UU. y Países Bajos). A su vez, China se erige como el 
principal mercado para la fabricación de bienes en el exterior.

- Uso de múltiples estrategias de entrada en mercados extranjeros, predominando la exportación de servicios realizados en Canarias (49,1\%), la prestación de servicios mediante empresa conjunta con un socio local $(36,8 \%)$ y la prestación de servicios en colaboración con socios locales $(29,8 \%)$.

- Numerosas y diversas barreras a la internacionalización identificadas por las propias empresas, destacando sobre todo la escasez de recursos y, en menor medida, las barreras gubernamentales en origen y las barreras comerciales.

- Relativa participación en programas públicos de apoyo a la expansión. El 38,6\% de la muestra ha participado en algún programa: Canarias Aporta (58,7\%), ICEXNext (37,0\%) o PIPE $(19,6 \%)$.

\section{La persona que asume la decisión de internacionalización en la empresa}

El 91,5\% de las personas a quienes concierne la decisión de internacionalización en las empresas encuestadas declara estar interesado en el desarrollo de algún tipo de actividad internacional, incluyéndose entre estas la importación y la exportación. Entre los aspectos que caracterizan a estos decisores pueden destacarse los siguientes:

- Son en su mayoría hombres $(68,3 \%)$, de entre 46 y 55 años (39,4\%), representando las mujeres solo el $31,7 \%$ del colectivo y situándose principalmente entre los 36 y 45 años de edad (32,6\%).

- Desempeñan este rol de decisor en temas relativos a la internacionalización de la empresa desde su puesto de propietario de la empresa $(64,1 \%)$, director/a o gerente
$(29,4 \%)$ o miembro de la alta dirección $(11,1 \%)$

- La mayor parte cuenta con estudios universitarios $(78,5 \%)$ (tabla 2$)$, destacando que es mayor el porcentaje de mujeres $(45,7 \%)$ que ha realizado parte de esta formación en el extranjero frente al registrado por los hombres $(31,3 \%)$.

Tabla 2. Decisores según nivel de estudios (en porcentaje)

Nivel de estudios finalizado

\begin{tabular}{lc}
\multicolumn{1}{l}{ Nivel de estudios finalizado } & $\%$ \\
Primaria/ E.G.B./ Secundaria Obligatoria & 0,7 \\
\hline $\begin{array}{l}\text { Bachillerato/ Formación profesional/ } \\
\text { Ciclos formativos }\end{array}$ & 18,0 \\
\hline $\begin{array}{l}\text { Diplomatura/ Grado/ Licenciatura/ } \\
\text { Ingeniería/ Arquitectura/ Máster }\end{array}$ & 78,5 \\
\hline Doctorado & 2,8 \\
\hline Total & 100,0 \\
\hline
\end{tabular}

- En el conocimiento de idiomas destaca el inglés hablado con fluidez por el $54,4 \%$ de estos decisores, seguido del alemán $(35,1 \%)$, francés $(27,0 \%)$, italiano $(23,4 \%)$ y portugués $(14,3 \%)$, identificándose que la mujer tiene mejor nivel de inglés que el hombre.

Al comparar a mujeres y hombres decisores con respecto a su trayectoria profesional, el estudio revela que:

- Las mujeres decisoras presentan menor antigüedad en la empresa (media: 8,7 vs 12,7 años); son menos emprendedoras (media: 1,2 vs 2,3 empresas creadas); tienen menor experiencia en impulsar proyectos innovadores (media: 5,3 vs 23,8 proyectos); $y$ se apoyan más en redes sociales en su actividad profesional (media: 3,89 vs 3,58 , en una escala de 1 a 5).

- Es mayor la proporción de mujeres que ha residido en el extranjero $(21,7 \%)$ que la de hombres $(10,1 \%)$, pero es menor la proporción de éstas que realiza viajes frecuentes al extranjero por motivos laborales $(34,8 \%$ frente a $64,6 \%)$ (figura 2$)$. 
Figura 2. Movilidad profesional del decisor según género

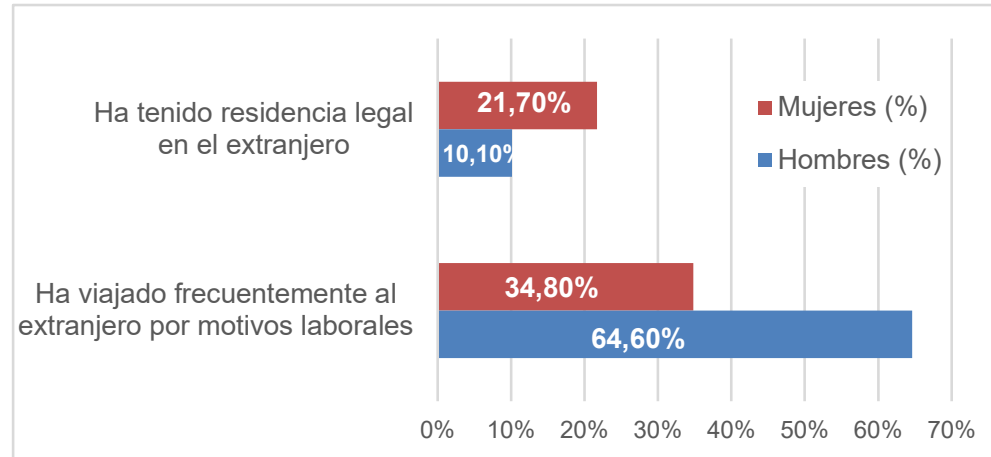

- Las mujeres perciben en mayor medida que los hombres la existencia de estereotipos sociales sobre roles de género $(50 \%$ vs $13,2 \%)$, así como discriminación por género en el ámbito laboral $(21,7 \%$ vs $8,1 \%)$. De hecho, 19 personas $(12,8 \%)$ han indicado haber sufrido discriminación, mayoritariamente por razón de género (figura $3)$.

Figura 3. Apoyo institucional para conciliación, estereotipos y discriminación por género en el ámbito laboral

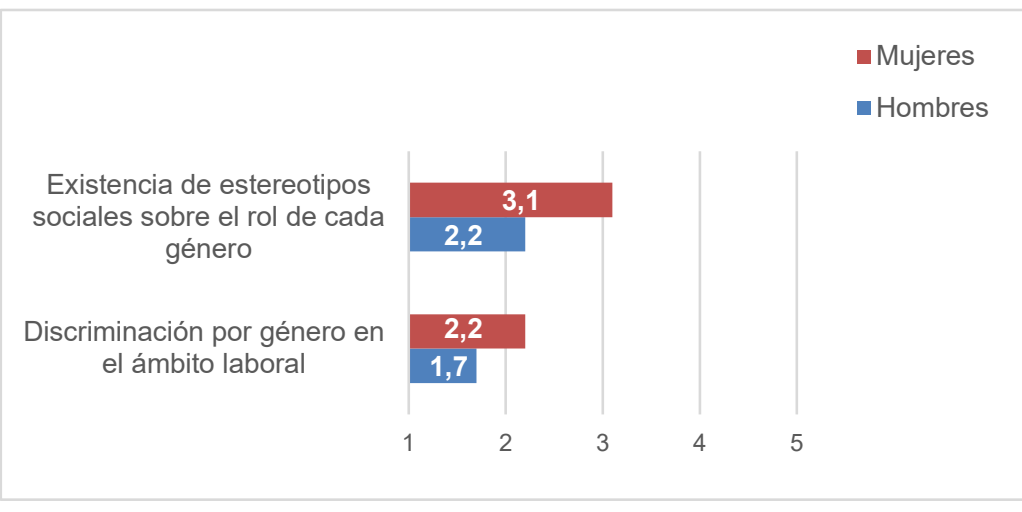

Finalmente, y bajo esta perspectiva de género, se analizó la posible relación entre el género del decisor y las características de la empresa en la que trabaja, encontrándose que (figura 4):

- La mujer trabaja, por lo general y en comparación con los hombres, en empresas más pequeñas. De hecho, los hombres trabajan en empresas donde la facturación se sitúa en torno a los $375.000 € /$ año, mientras que en las mujeres ronda los $250.000 €$.

- Con relación al sector de actividad, la proporción de mujeres decisoras en temas de internacionalización es mayor en la industria manufacturera $(64,3 \%$ de las decisoras son mujeres), en el sector agrícola (40,0\%) y en el de servicios $(39,3 \%)$.

- En cuanto a la estructura de gobierno de las empresas, existen diferencias significativas, ya que el tamaño medio del consejo de administración es de 3,3 personas en empresas con mujer decisora en temas de internacionalización y de 5,6 personas cuando este rol lo desempeña un hombre. A su vez, los hombres decisores trabajan en empresas con una media de 4,6 propietarios, mientras que las mujeres lo hacen en empresas con 1,7 propietarios por término medio.

- Respecto a la presencia de la mujer en diferentes niveles jerárquicos, este estudio encuentra que, en comparación con su contraparte masculina, las mujeres decisoras en la internacionalización trabajan en empresas donde es mayor la proporción de propietarias, consejeras y directivas.

\section{Género del decisor y actividad internacional de la empresa}

En este último apartado de los resultados se aborda el impacto que el género del decisor puede tener en la actividad internacional de la empresa. Una primera aproximación a esta relación se ha llevado a cabo nuevamente a través de la comparación, entre mujeres y hombres (ej. tests estadísticos de diferencias de medias), de diversas variables concernientes a la internacionalización de las empresas. Los resultados muestran que las empresas con decisoras mujeres solicitan ayuda pública para acometer la expansión exterior en similar medida que las empresas con decisores varones. 
Figura 4. Tamaño de la empresa, estructura de gobierno y presencia de la mujer en la empresa por género del decisor

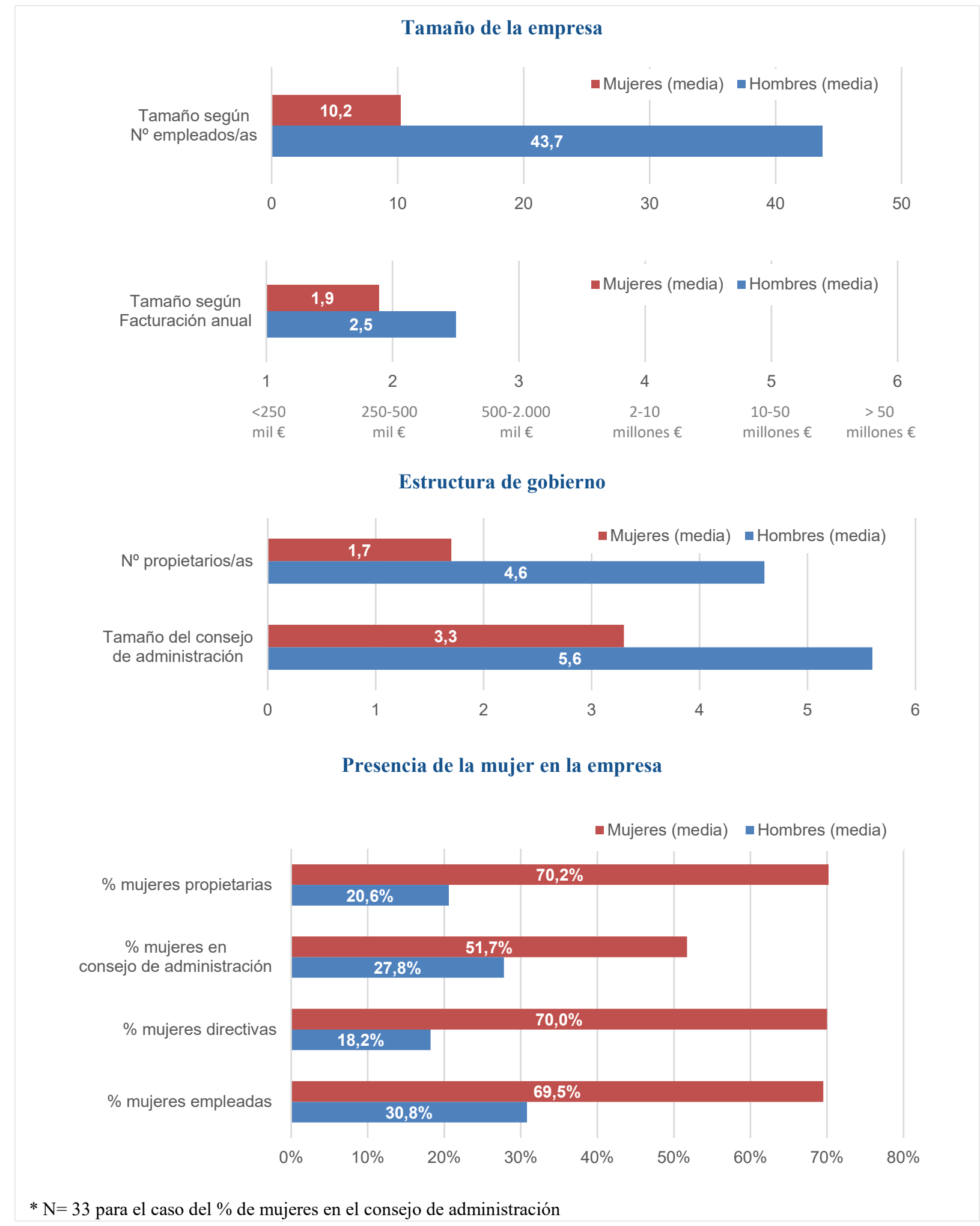

Ahora bien, las mujeres trabajan, por lo servicios en el extranjero $(54,2 \%$ vs $75,9 \%)$ y que general, en empresas que llevan menos años afrontan mayor dificultad para operar en el internacionalizadas (media: 7,9 vs 13,9 años), exterior (3,7 vs 2,3, en una escala de 1 a 5). A que prestan o comercializan en menor proporción pesar de ello, el porcentaje de facturación de las 
empresas en mercados exteriores (escala) o el número de países a los que se exporta productos/servicios (alcance) es similar para mujeres y hombres decisores, de forma que ambos alcanzan niveles semejantes de rendimiento de sus empresas en el exterior.

En segundo lugar, el impacto que el género del decisor puede ejercer en la internacionalización de sus empresas se ha estimado a través de tres modelos multivariantes (ver tabla 3). Con estas estimaciones se ha pretendido identificar cuáles son los factores que condicionan el interés por la expansión exterior de la empresa, considerándose entre ellos el género. Los resultados revelan que, entre las características del decisor con mayor influencia (tabla 3, Modelo 1), se encuentran aquellas que se adquieren con tiempo y esfuerzo, en particular el capital humano (conocimiento de idiomas y experiencia emprendedora) y social (amplitud de la red de apoyo) del decisor, no siendo su género determinante a este respecto.

Tabla 3. Factores que condicionan el interés en la internacionalización $(N=145)$

\begin{tabular}{|c|c|c|c|}
\hline Variables & $\begin{array}{c}\text { Modelo } 1 \\
\text { B }\end{array}$ & $\begin{array}{c}\text { Modelo } 2 \\
\boldsymbol{\beta}\end{array}$ & $\begin{array}{c}\text { Modelo } 3 \\
\text { 及 }\end{array}$ \\
\hline Constante & $-1,611$ & 1,487 & $-0,997$ \\
\hline \multicolumn{4}{|l|}{ Variables de control } \\
\hline Género $($ hombre $=0$, mujer $=1)$ & 0,042 & $-0,742$ & $-0,615$ \\
\hline Edad del decisor & $-0,016$ & $-0,053$ & $-0,054$ \\
\hline Antigüedad empresa & 0,000 & 0,001 & 0,003 \\
\hline \multicolumn{4}{|l|}{ Variables del decisor } \\
\hline $\mathrm{N}^{\mathrm{o}}$ países estudio & 0,283 & & 0,091 \\
\hline Conocimiento de idiomas & $0,133 *$ & & 0,071 \\
\hline Emprendedor & $1,317^{*}$ & & 0,903 \\
\hline Amplitud de sus redes & $1,058 *$ & & $1,562 *$ \\
\hline Fortaleza de sus redes & 0,123 & & $-0,374$ \\
\hline Disponibilidad para viajar & 0,260 & & 0,184 \\
\hline Disponibilidad para residir extranjero & $-0,236$ & & $-0,246$ \\
\hline \multicolumn{4}{|l|}{ Variables de la empresa } \\
\hline Nivel tecnológico & & $\mathbf{0 , 5 8 8} * *$ & $0,560 *$ \\
\hline Peso de la facturación digital & & 0,015 & 0,018 \\
\hline Proporción de mujeres en el equipo directivo & & $\mathbf{0 , 0 2 9} * *$ & $\mathbf{0 , 0 3 4 * *}$ \\
\hline $\mathbf{R}^{2}$ de Nagelkerke (\%) & $19,8 \%$ & $29,3 \%$ & $39,3 \%$ \\
\hline Chi-cuadrado Modelo [g.l.] & $18,674 *[10]$ & $25,246 * * *[6]$ & $35,034 * *[13]$ \\
\hline Chi-cuadrado Bloque [g.l.] & $17,842 *[7]$ & $22,103 * * *[3]$ & $18,716 * * *[3]$ \\
\hline Porcentaje correcto de predicciones & $83,4 \%$ & $84,1 \%$ & $89,4 \%$ \\
\hline
\end{tabular}

$* \mathrm{p}<.05, * * \mathrm{p}<.01, * * * \mathrm{p}<.001$ 
Los resultados también señalan que el interés en la actividad internacional puede venir determinado por características de las propias empresas (tabla 3, Modelo 2), especialmente su nivel de base tecnológica. De esta forma, el uso intensivo de tecnología y conocimiento también incrementa el interés de los decisores de la empresa por su internacionalización. Además, cabe resaltar el impacto positivo que el peso de las mujeres en los equipos directivos genera en el interés de las empresas canarias por la internacionalización empresarial.

El Modelo 3 en tabla 3, que incluye la totalidad de las variables en la estimación confirma que, si bien el género del decisor no condiciona el interés de la empresa por acometer actividades internacionales, la mayor proporción de mujeres en el equipo directivo de las empresas sí lo hace y de forma positiva. Este hallazgo es de gran importancia porque justifica, más allá de una perspectiva de equidad o justicia social, el interés económico que tiene la incorporación de la mujer a los puestos relevantes de la jerarquía organizativa de las empresas.

\section{Conclusiones}

Pese al creciente interés en estudiar la igualdad de género en el ámbito de la empresa, son escasos los datos, estudios o artículos que analizan la participación de la mujer en proyectos de internacionalización empresarial o incluso su presencia en empresas con potencial para dicha internacionalización. Esta falta de información es aún más evidente en el caso de Canarias, por lo que el presente estudio representa un primer paso para cubrir este importante vacío.

$\mathrm{Si}$ bien diversos resultados hallados en materia de género e internacionalización sobre Canarias son concordantes con los obtenidos por Núñez Varo (2021) en su análisis de las empresas españolas que han tenido relación con el ICEX en el período 2003-2019 (ej. las empresas lideradas por mujeres suelen tener menos ingresos y empleados, así como contratar preferiblemente a mujeres), la gran mayoría de las variables analizadas en nuestro estudio no encuentran precedentes en informes previos realizados en España. Por ejemplo, el estudio realizado aborda el papel y características de la mujer en el ámbito de los negocios internacionales a partir de su comparativa con el hombre; el trabajo aporta información primaria no incluida en las bases de datos secundarias disponibles (ej. estereotipos y discriminación laboral, conciliación trabajo-familia, porcentaje de facturación a través de canales digitales); finalmente, se recaba también información de empresas con diferentes actividades internacionales, así como de aquellas otras con y sin interés por acometer la internacionalización, lo que permite su comparación y la obtención de hallazgos de interés para los decisores públicos.

A tenor de la metodología empleada y los resultados alcanzados, y con el propósito de favorecer el desarrollo de programas que promuevan la participación de mujeres y hombres en proyectos de internacionalización en la empresa canaria, se recomienda crear un observatorio de la actividad internacional de la empresa en Canarias, de utilidad para recabar información puntual y actualizada sobre los retos a los que se enfrentan las empresas dirigidas por mujeres y dirigidas por hombres que tienen potencial internacional y aún no se encuentran internacionalizadas, así como profundizar en el análisis de los sectores que registran un menor grado de internacionalización y que pueden favorecer la creación de empleo, así como mejorar la competitividad y la diversificación de la economía canaria si afrontasen el reto de la internacionalización.

\section{Referencias}

Eden, L., \& Gupta, S. F. (2017). Culture and context matter: gender in international business and management. Cross Cultural \& Strategic Management, 24(2), 94-210.

Naciones Unidas. (2015). Transformar nuestro mundo: la Agenda 2030 para el Desarrollo Sostenible, Resolución aprobada por la Asamblea 
General el 25 de septiembre de 2015. Disponible en http://www.un.org/es/comun/docs/?symbol=A/RES/ 70/1 [fecha de consulta: 22.12.2021].

Núñez Varo, J. M. (2021). La internacionalización empresarial a través de la lente de género. Un análisis del apoyo de ICEX con perspectiva de género. ICEX. Disponible

en

http://www.revistasice.com/index.php/ICE/article/vi ew/7274 [fecha de consulta: 30.07.2021]. 